\title{
Cluster size entropy in the Axelrod model of social influence: small-world networks and mass media
}

\author{
Yérali Gandica ${ }^{1}$ \\ Instituto Venezolano de Investigaciones Científicas. \\ Centro de Física, Altos de Pipe, Carretera Panamericana, Km 11, \\ Caracas 1020A, Venezuela.
}

\author{
A. Charmell, J. Villegas-Febres \\ Departamento de Estado Sólido, Instituto de Física, \\ Grupo de Quimicofísica de Fluidos y Fenómenos \\ Interfaciales (QUIFFIS), Departamento de Química, \\ Universidad de Los Andes, Mérida 5101, Venezuela \\ I. Bonalde \\ Departamento de Física, Universidad Simón Bolivar, \\ Valle de Sartenejas, Baruta, \\ Apartado Postal 89000, Caracas 1080-A, Venezuela.
}

October 26, 2018

\begin{abstract}
We study the Axelrod's cultural adaptation model using the concept of cluster size entropy $S_{c}$ that gives information on the variability of the cultural cluster size present in the system. Using networks of different topologies, from regular to random, we find that the critical point of the wellknown nonequilibrium monocultural-multicultural (order-disorder) transition of the Axelrod model is unambiguously given by the maximum of the $S_{c}(q)$ distributions. The width of the cluster entropy distributions can be used to qualitatively determine whether the transition is first- or second-order. By scaling the cluster entropy distributions we were able to obtain a relationship between the critical cultural trait $q_{c}$ and the number $F$ of cultural features in regular networks. We also analyze the effect of the mass media (external field) on social systems within the Axelrod model in a square network. We find a new partially ordered phase whose largest cultural cluster is not aligned with the external field, in contrast with a recent suggestion that this type of phase cannot be formed in regular networks. We draw a new $q-B$ phase diagram for the Axelrod model in regular networks.
\end{abstract}

${ }^{01}$ Correspondence author. E-mail: ygandica@gmail.com 


\section{Introduction}

Recently, research in complex systems has paid particular attention to elucidate some of the mechanisms leading to interesting economic and social phenomena, such as opinion formation, self-organization, distribution of richness, formation of coalitions, land and air traffics, and evolution of social structures [1, 2, 3]. Several theoretical approaches have been proposed to understand social systems. Axelrod 44 introduced a model to study in particular the dissemination of cultures among interacting individuals or agents in which a) the more culturally similar are the agents, the greater the chance of interaction between them, and b) interaction increments similarity between individuals. Among the interesting results obtained with this model is a nonequilibrium transition from a monocultural state, where all agents share the same cultural features, to a multicultural state, where individuals mostly have their own features, as the cultural diversity increases [5].

The Axelrod model has been widely employed to analyze the effect of cultural drift caused by noise [6, 7, 8, repulsive interactions between individuals 9 , and mass media [10, 11, 12, 13, 14] on social systems. The effect of the mass media, which is normally modeled as a uniform external field with values in the range $[0,1]$, has been of special interest since it was discovered that in finite square networks there is a critical field $B_{c}=0.05$ above which the state of the system is always multicultural (disordered) [10. It was also found that for values of the 
number of cultural traits $q>q_{c}$ the state is always multicultural, independently of the field value $B$ [10. Here, $q_{c}$ is the critical value of the nonequilibrium multicultural-monocultural phase transition in the absence of an external field $(B=0)$. Later, it was shown that even for vanishing $B$ the monocultural state is destabilized in very large systems [14]. More recently, it was found that for the Axelrod model in complex networks the system can order in a vector state different from the one imposed by the external field [12]. All these are very counterintuitive findings, opposed to what is classically found in physics for spin systems that monotonously align with the external field. Interestingly, ordered phases that are not aligned with the external field are only encountered in fully connected, in random and in scale-free networks. It was thus claimed that longrange interactions, absent in regular lattices, are required for the appearance of this result 12 .

The nonequilibrium phase transition (hereafter we will omit the term "nonequilibrium") of the Axelrod model is characterized by an order parameter $\phi$ that is usually defined as the average size of the largest cultural cluster $C_{\max }$ in the system normalized by the total number of agents $N ; \phi=C_{\max } / N$. In the monocultural (ordered) state $\phi \rightarrow 1$ and in the multicultural (disordered) state $\phi \rightarrow 0$. Even though this order parameter appropriately identified both the ordered and the disordered phase emerging in the Axelrod model, it does not clearly define the critical region. Here, we show that the cluster size entropy 
$S_{c}$, which is defined in terms of the probability that an occupied site of the lattice belongs to a cluster containing $s$ sites, can be used as another powerful tool for the analysis of the phenomenology of the Axelrod model. The cluster size entropy measures the number of clusters of different sizes and is related to the diversity of the system [15. Theoretically, as a function of the probability of occupation $S_{c}$ should be zero in both the ordered and the disordered phase, since the former is constituted by a single cluster of the size of the system and the latter is formed by a large number of small clusters of similar sizes. $S_{c}$ should have a maximum at the transition where the diversity of cluster sizes is maximum. Thus, a peak develops as the phase transition takes place, which leads to a much better definition of the critical region.

In statistical physics, cluster size entropy has been used in the study of problems such as percolation [16, 17, 15, 18, and complex systems [9, 19, 20, 15, 18. In their studies on percolation, Tsang and co-workers [15, 18, found that the cluster entropy shows a maximum at the percolation threshold, where a group of neighboring occupied sites forms a cluster that expands from one edge of the 2D lattice to the opposite one causing an abrupt decrease in the cluster entropy of the system. In the context of the Axelrod model, cluster entropy measures the number of cultural groups of different sizes and was first used by Villegas-Febres and Olivares-Rivas 21] in an attempt to establish a connection with thermodynamics. Cluster entropy was also utilized within the 
Axelrod model to partially characterize the inclusion of repulsion among agents in a regular lattice 9 .

To demonstrate the usefulness of the cluster entropy in the analysis of complex networks, here we employ this property for the first time to carry out a throughout study on the monocultural-multicultural phase transition of the Axelrod model. We analyze the effects of the topology of the network and the mass media on this phase transition. Regarding the topology, we vary the probability $p$ of random rewiring between sites from 0 (regular networks) to 1 (random networks). In addition to determining more exactly the critical value $q_{c}$ of the Axelrod model, we establish a mathematical expression that relates $q_{c}$ with the important parameter $F$ (number of cultural features) in finite regular lattices and display in a much clearer form some other known properties. Considering the imposition of the mass media, we find that partially ordered states that are not aligned with the field can be formed in short-range-interaction regular networks, in contraposition to the claim by González-Avella et al. [12]. A new $q-B$ phase diagram is proposed for the Axelrod model in regular lattices.

\section{Axelrod model}

The original Axelrod model is defined on a square lattice of $N$ sites (social agents). The state of the $i^{t h}$ agent is defined by a set of $F$ cultural features (e.g., religion, sport, politics, etc.) represented by a vector $C_{i}=\left(C_{i 1}, C_{i 2}, \ldots, C_{i F}\right)$. Each feature $C_{i k}$ of the agent $i$ is first randomly assigned with a uniform dis- 
tribution of the integers in the interval $[0, q-1]$. The variable $q$ defines the cultural traits allowed per feature and thus measures the cultural variability in the system. There are $q^{F}$ possible cultural states.

The procedure to establish the dynamics of the system is as follows: (1) Choose randomly two nearest neighbor agents $i$ and $j$, then (2) calculate the number of shared features (cultural overlap) between the agents $\ell_{i j}=\sum_{k}^{F} \delta_{C_{i k}, C_{j k}}$. If $0<\ell_{i j}<F$ then (3) pick up randomly a feature $k$ such that $C_{i k} \neq C_{j k}$ and with probability $\ell_{i j} / F$ set $C_{i k}=C_{j k}$. These time steps are iterated and the dynamics stops when a frozen state is reached; i.e., either $\ell_{i j}=0$ or $\ell_{i j}=F, \forall i, j$. A cluster is a set of connected agents with the same state. Monocultural or ordered phases are composed of a cluster of the size of the system where $\ell_{i j}=F, \forall i, j$. Multicultural or disordered phases consist of two or more clusters.

To study the effect of an external field in the original Axelrod model just described some modifications are needed. We define a uniform external field as a vector $M=\left(m_{1}, m_{2}, \ldots, m_{F}\right)$, where $m_{n} \in[0,1, \ldots, q-1]$, with strength $B \in$ $[0,1]$. This parameter $B$ regulates the probability for the agent-field interactions. Each agent has a probability $B$ of interacting with the field and a probability $(1-B)$ of interacting with one of its nearest neighbors.

In the dynamic described above the agent $j$ is substituted by the field $M$ and the whole sequence follows in the same way: (1) Choose randomly an agent $i$, then (2) with probability $B$ agent $i$ and the field $M$ interact, (3) calculate 
the number of shared features (cultural overlap) between the agent and the field $\ell_{i M}=\sum_{k}^{F} \delta_{C_{i k}, M_{n}}$. If $0<\ell_{i M}<F$ then (4) pick up randomly a feature $k$ such that $C_{i k} \neq m_{k}$ and with probability $\ell_{i M} / F$ set $C_{i k}=m_{k}$. If agent $i$ and the field $M$ do not interact, (5) choose randomly an agent $j$ in the nearest neighborhood of agent $i$. If with probability $1-B$ agents $i$ and $j$ interact, (6) compute the cultural overlap $\ell_{i j}=\sum_{k}^{F} \delta_{C_{i k}, C_{j k}}$. If $0<\ell_{i j}<F(7)$ with probability $\ell_{i j} / F$ set $C_{i k}=C_{j k}$. The dynamics stops when a frozen state is reached.

To generate randomized lattices, required for the topology analysis, we used the Watts-Strogatz algorithm [22. Starting with a regular network of $N$ agents (with periodic boundary conditions), each link is visited and with probability $p$ is removed and rewired at random (avoiding self-linked nodes). This random rewiring process produces networks with topologies that go from perfect regularity $(p=0)$ to full randomness $(p=1)$.

\section{3 cluster entropy}

The cluster size entropy is defined as [15]

$$
S_{c}(P)=-\sum_{s} W_{s}(P) \ln W_{s}(P)
$$

where $P$ is the probability of occupation (probability $1 / q$ of taking a particular value of the cultural trait) and $W_{s}(P)$ the probability that an agent belongs to a cluster of size $s$. 

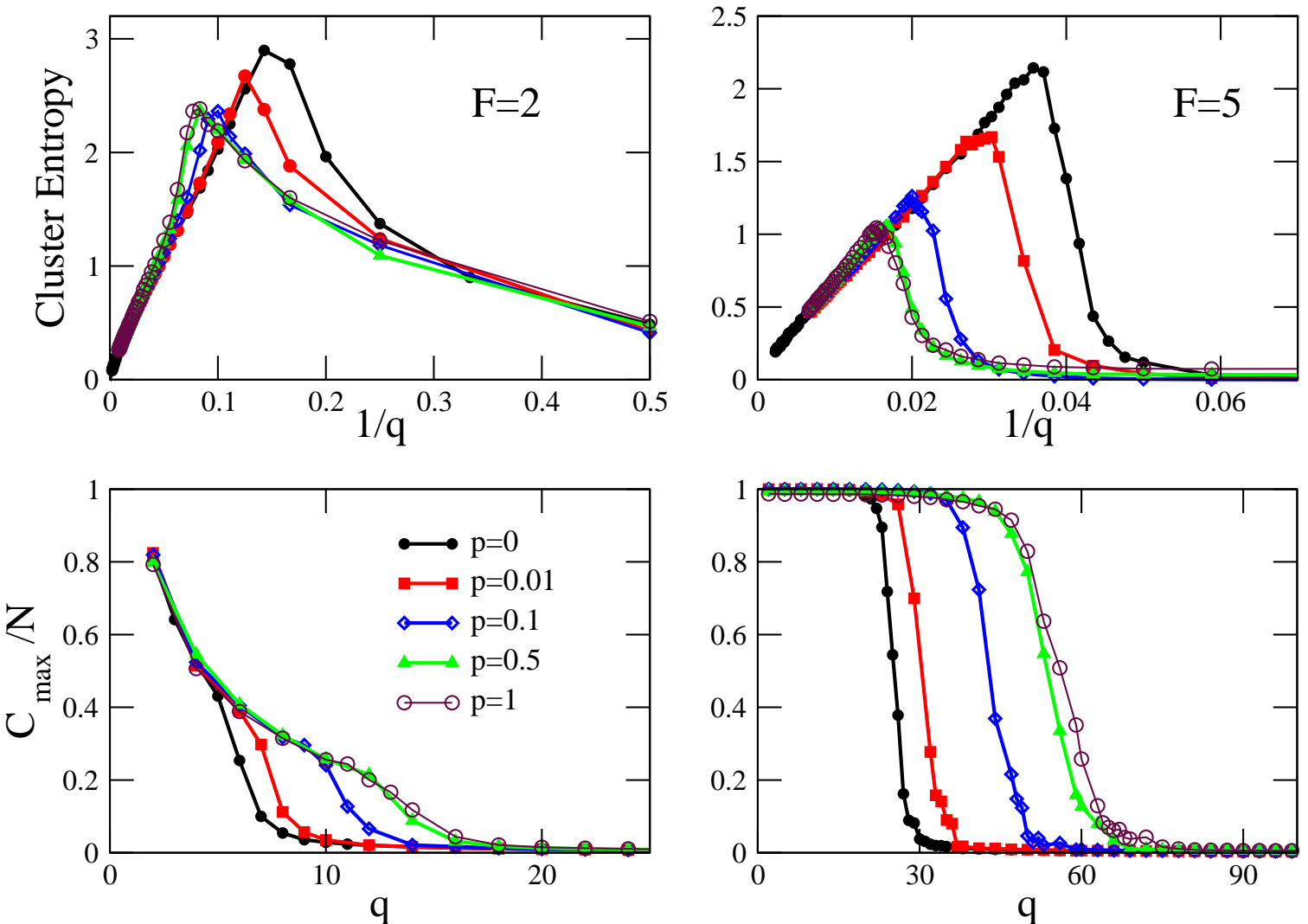

Figure 1: (Color online) Cluster size entropy as a function of the probability of occupation $1 / q$ and order parameter versus $q$ for $F=2$ and $F=5$ in networks of $40 \times 40$ with disorder parameter $p$ equals $0,0.01,0.1,0.5$ and 1 . The peak of the cluster entropy distributions occurs at the onset of the phase transitions as characterized by the order parameter $\phi$ (see text).

\section{4 network topology in the Axelrod model}

Here we vary the disorder parameter $p$ of the network from 0 to 1 to see the

effect on the monocultural-multicultural phase transition of the Axelrod model.

Figure 1 displays the cluster size entropy as a function of the probability of occupation $P=1 / q$ and, for comparison, the order parameter $\phi$ against the cultural trait $q$ for $\mathrm{F}=2$ and $\mathrm{F}=5$ in networks of $N=40 \times 40$ agents. There are various relevant issues in this figure that need consideration. The maximum 
of the cluster entropy curves is located around the corresponding onset trait $q_{c}$ of the monocultural-multicultural phase transitions as studied with $\phi$. The peak of a cluster size entropy distribution corresponds to the state of maximum size disorder that occurs at the transition. Thus, the average size of the largest cluster becomes finite when the maximum size disorder occurs. The size entropy brings out the fact that as the system moves from a disordered phase, in which most agents occupy each a single cluster, to an ordered phase, in which all agents occupy just one cluster of the system size, the system goes through a critical region where agents become agglomerated in clusters of different sizes. We see in the curves of Fig. 1 that the maximum of the cluster entropy defines more precisely the critical value $q_{c}$ than the finite value of the order parameter $\phi$.

Another feature to observe in Fig. 1 is that the critical value $1 / q_{c}\left(q_{c}\right)$ becomes smaller (larger) as $p$ goes from 0 (regular networks) to 1 (fully random networks), in complete agreement with the results obtained with the order parameter $\phi$ by Klemm et al. 23. We believe that the collapse of the curves corresponding to $p>0.5$ is due to finite-size effects and not to the lack of any further dynamics that indeed seems to develop up to $p=1$ in networks of large size 23.

Next, we point out that the height of the cluster entropy curves in Fig. 1 1 reveals a new characteristics of the Axelrod model that was impossible to uncover by means of the order parameter $\phi$. As $p$ increases the maximum of the size 


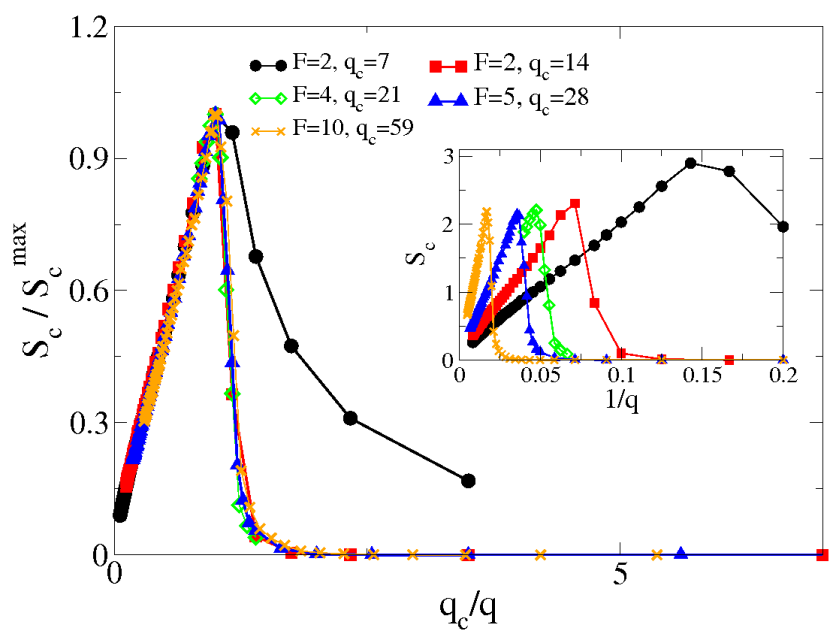

Figure 2: (Color online) Normalized cluster size entropy $S_{c} / S_{c}^{\max }$ against the normalized probability of occupation $q_{c} / q$ for a network of disorder $p=0$ and size $40 \times 40$ for different values of $F$. The data suggest that the Axelrod model for $F=2$ is in a different universality class from that for $F>2$ (see text). The inset shows the regular data.

entropies becomes smaller, suggesting less cluster diversity at the transition.

Thus, it appears that in random networks, due to the presence of long-range links, the formation of the system-size cluster defining the ordered state of the Axelrod model is more efficient (explores a smaller region of the cluster size space) than in regular networks.

The most highlighted findings of the present analysis on the effect of topology in the Axelrod model are observed in the scaled data of Figs. 2, 3, and 4 for network randomness $p=0,0.1$ and 1 , respectively. These figures display the normalized cluster entropy $S_{c} / S_{c}^{\max }$ versus the normalized probability of occupation $q_{c} / q$ for different values of the cultural-feature number $F$. Here, $S_{c}^{\max }$ is the value of the cluster entropy at the peak maximum. The insets show the corresponding regular data. All three plots show that, independently of the 


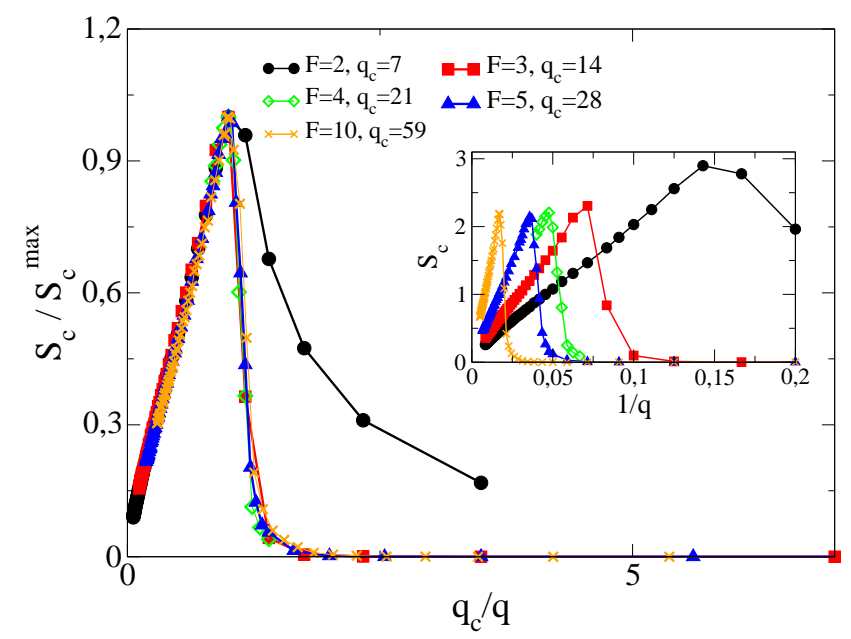

Figure 3: (Color online) Normalized cluster size entropy $S_{c} / S_{c}^{\max }$ against the normalized probability of occupation $q_{c} / q$ for a network of disorder $p=0.1$ and size $40 \times 40$ for different values of $F$. The inset shows the regular data.

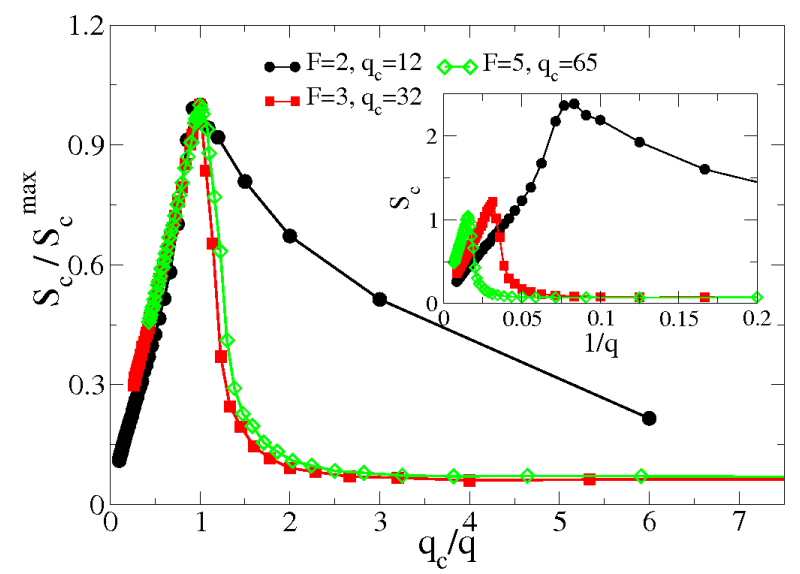

Figure 4: (Color online) Normalized cluster size entropy $S_{c} / S_{c}^{\max }$ against the normalized probability of occupation $q_{c} / q$ for a network of disorder $p=1$ and size $40 \times 40$ for different values of $F$. The inset shows the regular data. 
network randomness, the overall dynamics of the Axelrod model for $F=2$ is unambiguously different from that for any value $F>2$, and that the dynamics is the same for all values $F>2$. The plots indicate that the Axelrod model for $F=2$ and $F>2$ must belong to different "universality classes" in the terminology of statistical physics. This agrees with previous works that claimed that the transition is first-order type for $F>2$ and second-order type for $F=2$ [5, 6].

The width of the cluster size entropy distribution may give a clue of the order of the transition. The more narrow distributions of the cluster entropy for $F>2$ (Figs. 2, 3, and 4) compared with the distributions for $F=2$ indicate that the system crosses over from disorder (a large number of clusters exist) to order (just one cluster size is present) in a sudden manner, which is typical of first-order-like transitions. Broad distributions, instead, suggest that the system moves from one regime to the other in a smooth manner. A finite number of clusters of all sizes starts to form in the disordered phase and as the system crosses over to the ordered side those clusters of all sizes agglomerate in just one of the size of the system. This is the behavior expected in second-order-like transitions.

Notably, the normalized cluster entropies are independent of $F$ for $\left(q_{c} / q\right)<$ 1 , indicating that there is a unique Axelrod dynamics for values $q>q_{c}$. This conclusion is robust against finite-size effects, as is demonstrated by the data 
shown in Fig. [5,

It is widely known that the monocultural-multicultural phase transition occurs at a critical value $q_{c}$ that increases as $\mathrm{F}$ augments [5, 24]. However, there is no expression relating $q_{c}$ and $F$. Here, we provide such an equation valid in regular lattices $(p=0)$ of finite sizes:

$$
q_{c} \approx 7 *(F-1) .
$$

This expression 1) defines the value of the cultural trait $q$ above which the system will always be in a multicultural state and 2) states that for any value of $F$ the system will always be monocultural for $q<7$. Equation (2) appears to hold for other values of the network randomness $p$ with a pre-factor that becomes larger as $p$ increases.

\section{5 external field or mass media in the Axelrod model}

González-Avella et al. [12] found that in the presence of an external field the Axelrod model displays a rich $q-B$ phase diagram in fully connected, in random and in scale-free $2 \mathrm{D}$ networks for $F=10$. A second ordered phase was found that is not aligned with the external field and that in complex networks does not cover the whole system. González-Avella et al. argue that this ordered phase is caused by the long-range interactions characteristic of complex networks, and that such a phase does not exist in regular (short-range interaction) networks.

Figure [6] shows the cluster entropy against $1 / q$ and the order parameter 
versus $q$ in a $40 \times 40$ regular network for several values of the field strength $B$ and for $F=5$ and $F=10$. One of the effects of the external field is to move the critical value $1 / q_{c}$ toward higher occupation probabilities, as occurs when the number of cultural features $F$ is reduced (see Figs. 1 and 2). Interestingly, the limiting $\left(1 / q_{c}\right)=0.128$ as $B \rightarrow 1$ is very close to the limiting $\left(1 / q_{c}\right)=0.142$ as $F \rightarrow 2$.

More relevant, a second peak is observed in the cluster entropies of Fig. 6 for $0.2<B<0.8$ that indicates the occurrence of a second phase transition. These cluster entropies are constituted by two overlapping entropy distributions whose peaks correspond to the onset of phase transitions. The second peaks in $S_{c}$ in the upper panels of Fig. 6 correspond to reentrant behaviors in the order parameter $\phi$ in the lower panels. That is, as the value of $q$ is lowered the system moves from a multicultural to an ordered-like phase, then returns to the multicultural phase, and finally goes to a monocultural phase. The ordered-like phase makes the phase diagram of the Axelrod model in regular networks more complex than previously thought 12 . The appearance of the second transition both for $F=5$ and for $F=10$ indicates that its existence does not depend on $F$.

A further analysis reveals that the system in the extra ordered phase, called here "crossing phase", is mainly formed by a large cultural cluster whose state is not aligned with the external field. This crossing phase, whose order parameter 
$\phi<1$ (meaning that the largest cluster does not have the size of the system), is closely related to the "orthogonal" ordered phase reported by González-Avella et al. [12] of the Axelrod model in $50 \times 50$ random networks. Contrasting with the conclusions by González-Avella et al., our results indicate that long-range interactions are not fully required in the Axelrod model to form an ordered state whose orientation is not parallel to the external field.

In Fig. 7 we draw a new phase diagram of the Axelrod model in regular networks by using the peaks of the size entropy distributions for $F=5$. The highest peak at $q_{c}$ of each $S_{c}$ distribution in Fig. 6 agrees with the onset of the monocultural-multicultural transition in the corresponding $\phi$ curve. The second peak at $q^{*}$ in the entropy distributions is assigned to the multicultural-crossing transition. In the monocultural phase (colored red) the state $(\phi \sim 1)$ is aligned with the external field for $q<q_{c}$. In the crossing phase (colored yellow) the ordered state $(\phi<1)$ is not parallel to the external field for $q_{c}<q<q^{*}$ and $0.2<B<0.8$. In this region there is no overlap between the ordered state and the field. The multicultural phase (colored blue) is completely disordered. In particular, the state in regular networks for $F=5$ is always monocultural for $q<8$ and multicultural for $q>28$ independently of the external field, in agreement with Eq. (2).

Finally, in connection with the analysis of the previous section, we remark here that the data for $F=10$ in Fig. 6 provide another good example to show 
that the cluster size entropy can indicate better the critical value $q_{c}$ than the order parameter. One can see that for the cases $B=0.5$ and $B=0.7$ the transition onset is better determined by the second peak of the cluster size entropy than by the initial finite value of the order parameter, although this last property displays quite well the transition.

\section{Summary}

Thus far, the cluster size entropy has been hardly used in the analysis of phase transitions. Here, we showed that the cluster size entropy $S_{c}$ is a valuable tool that can be utilized as a complement of the order parameter $\phi$. Using the cluster entropy, we were able to both reproduce most of the results previously known for the Axelrod model in square networks and find new relevant results. We showed by a simple analysis that the Axelrod model for $F=2$ and $F>2$ belong to a different "universality" class. For regular lattices it was determined an expression that relates $q_{c}$ and $F$ and that defines the asymptotic values of the trait $q$ for the presence of multicultural and monocultural phases in the system.

We found a new partially ordered phase for the Axelrod model in regular lattices, in which the vector state of the largest cultural cluster is not aligned with the external field or mass media. This phase is similar to one previously reported for fully connected, scale-free and random networks, and leads to a new cultural trait-field $(q-B)$ phase diagram for the Axelrod model in regular networks. The results suggest that long-range interactions are not completely 
necessary for the existence of an ordered state that is not oriented along the applied field.

\section{7 acknowledgments}

J.V-F thanks CDCHTA for Grants C-1449-07-05-F and ADG-C-09-95. Y.G. acknowledges support from Misión Ciencia and IVIC doctoral fellowships. We thank computational support from Leonardo Trujillo at IVIC and Centro Nacional de Cálculo Científico (CeCalCULA) of the Universidad de Los Andes.

\section{References}

[1] P.W. Anderson, K. Arrow and D. Pines. The Economy as an Evolving Complex System. Addison-Wesley, Redwood, 1998.

[2] S. M.Oliveira,P. M. C.de Oliveira and D. Stauffer. Nontraditional Applications of Computational Statistical Physics. B. G. teubner. Stuttgart, 1999.

[3] C. Castellano, S. Fortunato and V. Loreto. Rev. Mod. Phys. 81, 591, 2009.

[4] R.Axelrod. J. Conflict Resolut. 41, 203, 1997.

[5] C. Castellano, M. Marsili and A. Vespignani, Phys. Rev. Lett. 85, 3536, 2000.

[6] K. Klemm, V. M. Eguíluz, R. Toral, and M. S. and Miguel, Physica A, 327, 1. 2003 
[7] K. Klemm, V.M. Eguíluz, R. Toral and M. S. Miguel. Phys. Rev. E, 67, 045101(R), 2003

[8] L. D. Sanctis and T. Galla, Phys. Rev. E, 79, 046108, 2009.

[9] A. Radillo-Díaz, L. A. Pérez, and M. del Castillo-Mussot, Phys. Rev. E. 80, 066107, 2009.

[10] J. C. González-Avella, M. G. Cosenza, K. Tucci. Phys. Rev. E, 72, $065102(\mathrm{R}), 2005$.

[11] J. C. González-Avella, V. M. Eguíluz, M. G. Cosenza, K. Klemm, J. L. Herrera and M. S. Miguel. Phys. Rev. E, 73, 046119, 2006.

[12] J. C. González-Avella, M. G. Cosenza, V. M. Eguíluz and M. S. Miguel. New J. Phys., 12, 013010, 2010.

[13] . A. H. Rodríguez and Y. Moreno. Phys. Rev. E, 82, 016111, 2010.

[14] L. R. Peres and J. F. Fontanari. J. Phys. A: Math. Theor., 43, 055003, 2010 .

[15] I. R. Tsang and I. J. Tsang. Phys. Rev. E, 60, 2684, 1999.

[16] D. Stauffer and A. Aharony. Introduction to Percolation Theory. Taylor and Francis, London. 1994, 2nd. ed.

[17] J. Hoshen and R. Kopelman. Phys. Rev. B, 14, 3438, 1976.

[18] I. J. Tsang, I. R. Tsang and D. V. Dyck. Phys. Rev. E, 62, 6004, 2000. 
[19] . M. A. F. Gomes, F. A. O. Souza and S. K. Adhikari. J. Phys. A, 28, L613, 1995.

[20] I. R. Tsang and I. J. Tsang. J. Phys. A, 30, L239, 1997.

[21] J. Villegas-Febres and W. Olivares-Rivas. Physica A, 387, 3701, 2008.

[22] D. Watts and S. H. Strogatz, Nature, 393, 440, 1998.

[23] K. Klemm, V. M. Eguíluz, R. Toral and M. S. Miguel. Phys. Rev. E, 67, 026120, 2003.

[24] K. Klemm, V. M. Eguíluz, R. Tora and M. S. Miguel. J. Econ. Dyn. Control, 69, 312, 2005 . 

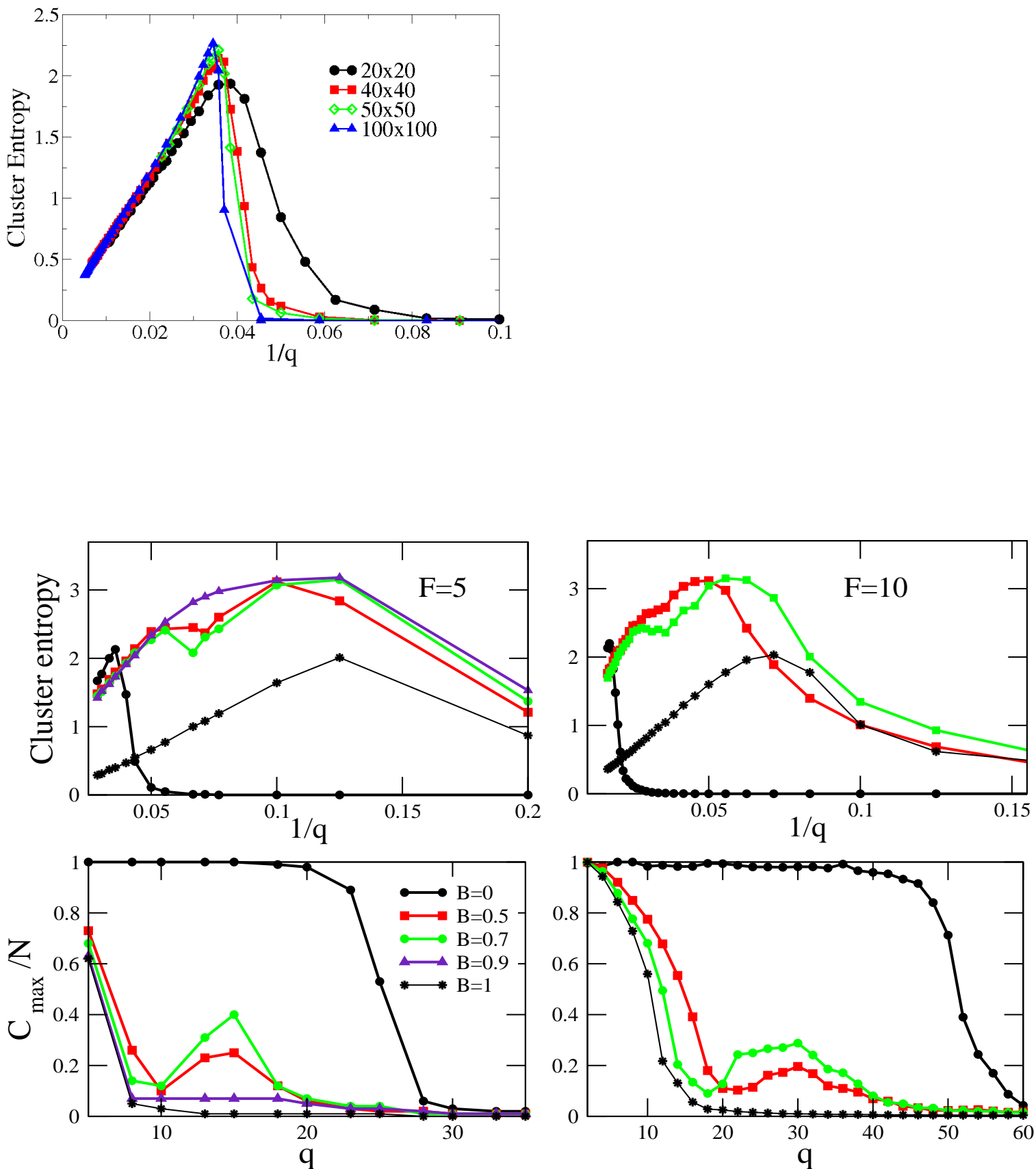

Figure 6: (Color online) Cluster entropy $S_{c}$ against $1 / q$ and order parameter $\phi$ as a function of $q$ for $F=5$ and $F=10$ in a $40 \times 40$ regular network for various field strengths $B$. For both values of $F$ a second transition develops in the field range $0.2<B<0.8$. For the sake of clarity the case $B=0.9$ not displaying a second transition is omitted in $F=10$. 


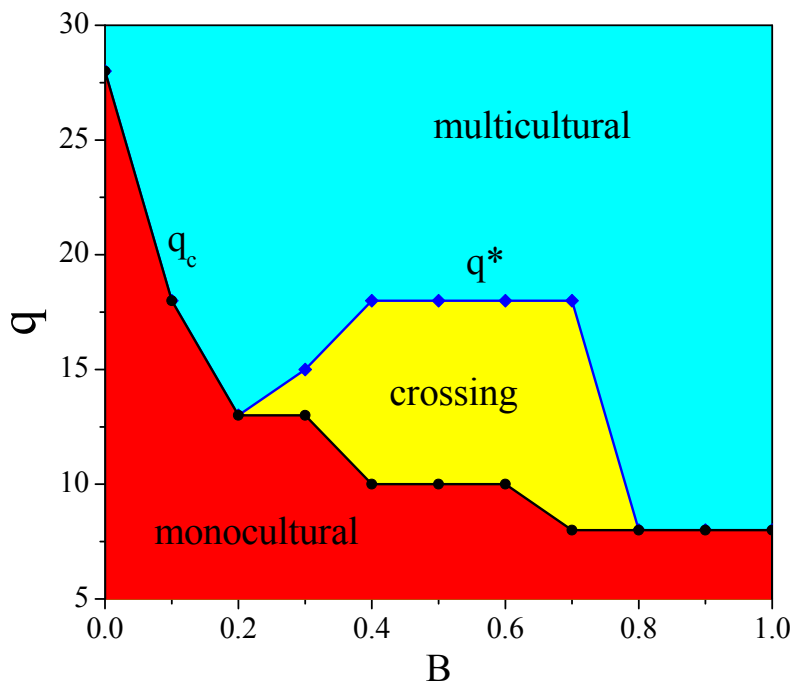

Figure 7: (Color online) $q-B$ phase diagram of the Axelrod model obtained for $F=5$ in a $40 \times 40$ regular network. Each point represents an average of 50-100 realizations. 